\title{
Affective Video Data Collection Using an Automobile Simulator
}

\author{
Tevfik Metin Sezgin and Peter Robinson \\ University of Cambridge \\ Computer Laboratory \\ Cambridge UK CB3 OFD \\ Metin.Sezgin@cl.cam.ac.uk \\ Peter.Robinson@cl.cam.ac.uk
}

\section{Introduction}

The correlation between driver status and safety has increased interest in automated systems that can infer the physical and mental state of drivers $[2,3]$. So far, most research on automated recognition of driver state has focused on physical state. More recently, researchers have proposed inferring the mental states of drivers by extracting drivers' facial expressions from video data. Although systems that infer physical state have been evaluated in simulators or in real driving conditions, this has not been the case for systems inferring mental states which rely primarily on video data. Consequently, issues regarding the design of an experimental setup to collect such affective data have not been explored. Here, we describe our experimental setup for collecting video data from drivers.

\section{Experimental Setup}

We used a state of the art car simulator to subject four drivers to three drives designed to trigger a number of driving-related mental states. Fig. 1 shows examples of the first driver showing confusion.
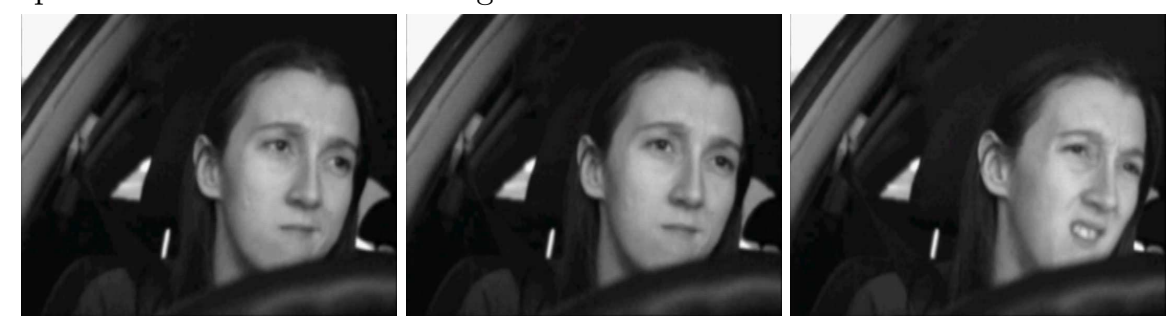

Fig. 1. Examples of the first driver expressing confusion.

The goal of our experiment was to observe how the drivers would behave when subjected to conditions intended to trigger complex mental states relevant to driving. Using the emotion taxonomy of Baron-Cohen et al [1], a set of complex mental states were chosen based on driving behaviour research. The chosen mental states were discomfort, uncertainty, nervousness, boredom, drowsiness, comfort, surprise, concentration, thinking. 


\section{Results}

To measure the effectiveness of our experimental setup in eliciting mental states, we administered a questionnaire and collected drivers' subjective evaluation of how they felt at different times of the trial. The questionnaires have revealed that our setup was successful in putting the drivers into bothered, upset, bored and drowsy mental states.

Our experiments also allowed us to gauge the utility of a car-simulator in collecting affective video data. Our analysis revealed a number of issues regarding the effectiveness our experimental setup and the challenges of collecting video data of good quality. For example, we found that certain events triggered stronger facial expressions compared to others. Also some conditions worked well for some subjects and drew unexpected responses from others. Our experiments also revealed the difficulty of capturing clear frontal views of the driver's face due to physical space limitations.

\section{Related and Future Work}

We collected affective data from drivers using a car simulator. Our evaluation focused on eight driving-related mental states and allowed us to explore the challenges in eliciting and collecting affective video data. The above aspects of our work collectively distinguish it from the existing related work including work by Lisetti and Nasoz [3] and Healey and Picard [2] who carried out similar experiments in real and simulated driving conditions, but mainly focused on the analysis of physiological data.

There are two main directions that we would like to explore. First, we would like to analyse the videos in more depth and annotate interesting parts to include the corresponding facial actions and mental states. We also we would like to investigate how the knowledge of drivers' mental state can be effectively used to facilitate driver-automobile communication, enhance road safety and the overall driving experience.

\section{Acknowledgements}

We would like to thank Dr. Rana el Kaliouby for making her mental state analysis software available to assess the quality of the face tracking. We also thank the TRL staff for their input in the design and execution of the data collection.

\section{References}

1. S. Baron-Cohen, O. Golan, S. Wheelwright, and J. J. Hill. London: Jessica kingsley publishers. Mind Reading: The Interactive Guide to Emotions., 2004.

2. Jennifer A. Healey and Rosalind W. Picard. Detecting stress during real-world driving tasks. IEEE Tran. on Intelligent Transportation Systems, 6-2:156-166, 2005.

3. Christine Laetitia Lisetti and Fatma Nasoz. Affective intelligent car interfaces with emotion recognition. In HCI 2005, Las Vegas, USA, Jul 2005. 